\title{
Concepciones de los estudiantes de un contexto rural sobre la fotosíntesis
}

\author{
Yobana Lucía Vega Páez ${ }^{1+}$, Nidia Yaneth Torres Merchán ${ }^{(1)}$, Eliana Yizeth Pedreros Benavides \\ Institución Educativa San Miguel, Universidad Pedagógica y Tecnológica de Colombia, Colombia
}

Autor de correspondencia: 1yobana.vega@uptc.edu.co Recibido: 26 de junio de 2020 Revisado: 30 julio de 2020

Aprobado: 29 de octubre de 2020 Publicado: 20 de noviembre de 2020

\section{Resumen}

La fotosíntesis es uno de los ejes temáticos más conflictivos para los estudiantes, por un lado, por su falta de comprensión y, por otro, es el tema con el cual más dificultades acumulan. Por ello, esta investigación pretende describir las concepciones acerca de la fotosíntesis de 24 estudiantes de grado décimo y undécimo de una institución educativa del municipio de Coper, en el contexto rural del departamento de Boyacá, Colombia. Se desarrolla según un enfoque cualitativo, que permite comprender cómo los estudiantes de un contexto rural entienden la fotosíntesis y qué otros conceptos pueden asociar en sus concepciones. Se utilizó un cuestionario de 30 preguntas. Se encontró que los estudiantes tienen ideas alternativas que a menudo los lleva a pensar que la fotosíntesis es un tipo de respiración de las plantas. También se hizo evidente que no reconocen en su totalidad los compuestos necesarios para que el proceso se lleve a cabo. Esto dificulta en gran medida la comprensión de conceptos como la nutrición autótrofa, la respiración, el intercambio gaseoso y rutas metabólicas.

Palabras claves: enseñanza de las ciencias, fotosíntesis, aprendizaje, medio rural

\section{(c) $)(1) \Theta \Theta$}

Para citar este artículo: Vega, Y., Torres, N., \& Pedreros, E. (2020). Concepciones de los estudiantes de un contexto rural sobre la fotosíntesis. Praxis \& Saber, 11(27), e11298. https://doi. org/10.19053/22160159.v11.n27.2020.11298 


\title{
Conceptions of students from a rural context about photosynthesis
}

\begin{abstract}
Photosynthesis is one of the most controversial topics for students, on the one hand, because of their lack of understanding and, on the other hand, it is a subject on which they have the most difficulties. Therefore, this research aims to describe the conceptions about photosynthesis of 24 10th and 11th grade students from an educational institution in the municipality of Coper, in the rural context of the department of Boyacá, Colombia. It was conducted according to a qualitative approach, which allows us to comprehend how students in a rural context understand photosynthesis and what other concepts they would associate in their conceptions. A 30-question questionnaire was used. It was found that students have alternative ideas that often lead them to think that photosynthesis is a type of plant respiration. It also became evident that they do not fully recognize the necessary compounds for the process to occur. This makes it very difficult for them to understand concepts such as autotrophic nutrition, breathing, gas exchange and metabolic pathways.
\end{abstract}

Keywords: science teaching, photosynthesis, learning, rural environment

\section{Concepções dos estudantes de um contexto rural sobre a fotossíntese}

\section{Resumo}

A fotossíntese é um dos eixos temáticos mais conflituosos para os estudantes, por um lado, devido à sua falta de compreensão e, por outro, é um tema com o qual acumulam mais dificuldades. Por esta razão, a pesquisa tem como objetivo descrever as concepções sobre a fotossíntese de 24 estudantes de décimo e décimo primeiro ano de uma instituição educacional no município de Coper, no contexto rural do departamento de Boyacá, Colômbia. Desenvolve-se de acordo com uma abordagem qualitativa, que permite compreender como os estudantes num contexto rural entendem a fotossíntese e que outros conceitos podem associar nas suas concepções. Foi utilizado um questionário de 30 perguntas. Verificouse que os estudantes apresentam ideias alternativas que muitas vezes os levam a pensar que a fotossíntese é um tipo de respiração das plantas. Também se evidenciou que eles não reconhecem completamente os compostos necessários para que o processo ocorra. Isto torna muito difícil a compreensão de conceitos como nutrição autotrófica, respiração, trocas gasosas e vias metabólicas.

Palavras-chave: ensino das ciências, fotossíntese, aprendizagem, ambiente rural

Este artículo hace parte de una investigación de trabajo de grado de maestría ${ }^{1}$ Se analiza cómo los estudiantes de un contexto rural "hablan ciencia" desde la experimentación del entorno natural para el aprendizaje del concepto de fotosíntesis. Por consiguiente, despertar el interés de los estudiantes para "hablar ciencia" es la necesidad que se encontró en las prácticas de aula de las ciencias naturales. Por ello, se busca acercarlos más a la condición necesaria del uso de un lenguaje científico.

1 Hablar ciencia desde la experimentación del entorno natural para el aprendizaje de la fotosíntesis. Trabajo de grado de Maestría en Educación. Universidad Pedagógica y Tecnológica de Colombia. 
A esto se suma la forma de llevar a cabo los procesos de educabilidad con los estudiantes desde la experimentación del medio para el fortalecimiento del concepto en mención, puesto que su participación en trabajos de campo tiene "un efecto motivador para generar razonamiento causal" (Torres \& Montenegro, 2018, p. 26). Estos procesos, señalan la importancia de que el aprendizaje desde las ciencias sea significativo. De allí, se infiere que al alumno le hace falta hablar sobre las experiencias y sobre las ideas que desde su entorno natural se pueden construir. Sin embargo, a pesar de que hace parte de su desarrollo, ellos no identifican con claridad las acciones que conlleva el término y muchas veces recitan los conceptos sin entenderlos y sin ver su aplicabilidad en situaciones de la vida cotidiana.

Desde esta mirada, nuestro interés es el análisis de concepciones en los estudiantes de un contexto rural, ya que sus experiencias de vida están articuladas a prácticas sociales asociadas al entorno natural. Por esta razón, es preciso abordar la ruralidad desde una perspectiva educativa, que nos permita reconocer esos escenarios que, junto con sus necesidades y perspectivas, nos lleven a dialogar acerca de la calidad educativa a través de la generación de conocimiento (Herrera \& Buitrago, 2015). Esto coincide con los estudios de Amiguinho (2011), quien afirma que "la escuela en el medio rural continúa dando muestras de la riqueza y del potencial de las experiencias educativas" (p. 26). No obstante, es evidente que desde el aula y desde la práctica docente "ha de hacerse visible [...] este enfoque como parte sustancial de la actualidad educativa" (Bustos, 2011 p. 156).

Con esto, se hace énfasis en que enseñar ciencia puede resultar tan difícil, no por la complejidad de sus contenidos, sino por la forma como esta se comunica a los estudiantes. Así, diversas investigaciones (Garnica \& Roa, 2013; Gómez, 2014; Macías, 2013; Sáenz, 2012) hacen su aporte en la enseñanza del concepto de la fotosíntesis al abordar ítems temáticos precisos para llevarlos al discurso de aula.

\section{La importancia del lenguaje científico}

Es importante reconocer que el lenguaje científico puede ser un proceso de difícil aprendizaje para los estudiantes, ya que no es el mismo que se utiliza en la cotidianidad. Como dice Lemke (1997), "el lenguaje no es solo vocabulario y gramática: es un sistema de recursos para construir significados" (p. 12). Por lo tanto, ese lenguaje va de la mano con la interrelación de conceptos e ideas, que sumadas permitirán la construcción de significados. Es decir, a través del lenguaje de la ciencia, los estudiantes, sin importar su nivel de escolaridad, pueden comprender la cultura científica.

Según Sanmartí (2007), "aprender ciencias comporta aprender a mirar y ver las experiencias desde puntos de vista distintos y a pensar sobre ellos desde concepciones que a menudo son diferentes de las intuitivas" (p. 2), más aún en este tipo de escenarios, porque esa interrelación con el medio logra concretar un nuevo pensamiento y un mirar dirigido a la idea concreta de un buen uso y alimentación de vocabulario. Ese lenguaje social especializado es el que se quiere abordar. Lemke (1997) da las pautas para abarcarlo desde el discurso de aula en las clases de ciencias.

En este caso particular, se analizan los discursos emitidos en el aprendizaje de la fotosíntesis. Macías (2013) afirma que "los contenidos relacionados con el proceso de la fotosíntesis son extensos y normalmente no se desarrollan en su totalidad, esto unido a lo abstracto y complejo que resulta el proceso" (p. 1). Es claro que bajo estas circunstancias 
el estudiante no logra una comprensión apropiada del tema en cuestión. Quizá por ello termina creyendo que la fotosíntesis y la respiración son lo mismo.

En esta medida, las investigaciones previas (Keleş \& Kefelib, 2010; Svandova, 2014) buscan solucionar esos inconvenientes, que se fundamentan principalmente en prescindir de las nociones disyuntivas que pueden aparecer a lo largo de los procesos de educabilidad, específicamente sobre los procesos de fotosíntesis y respiración.

Desde la perspectiva de Charrier et al. (2006), existen errores conceptuales al comprender ambos procesos - fotosíntesis y respiración-, y su génesis está directamente en el docente, en el diseño curricular y —era de esperarse- en los libros de texto; de allí que la revisión de los contenidos relacionados acerca de la fotosíntesis desde los textos escolares a lo largo de la historia (Astudillo \& Gene, 1984; Barrutia et al., 2016; González et al., 2003) determine que la caracterización de las concepciones de los estudiantes es errónea y que presenta serias dificultades.

A menudo, a partir de la forma como se enseñan los conceptos, los estudiantes tienden a pensar que la fotosíntesis es un proceso inverso a la respiración (Charrier et al., 2006). Hoy se evidencia que, para enseñar a los estudiantes temas complejos como estos, se ha de partir de las ideas o nociones que tienen acerca de ellos, con el fin de encontrar alternativas que faciliten su transformación.

Por su parte, es frecuente encontrar que las concepciones de los estudiantes acerca de la fotosíntesis (Gómez \& Velazco, 2015; Sáenz, 2012) difieren en gran medida con respecto a las concepciones que tienen sobre la nutrición de las plantas verdes (Angosto, 2018; Cañal, 1991), pues tienden a creer que son lo mismo y, no visibilizan el proceso que se desarrolla en conjunto. El conocimiento didáctico del contenido sobre la fotosíntesis por cuenta de los docentes (Garnica \& Roa, 2012) también tiene gran incidencia en los elementos que permiten construir y desarrollar la temática.

De la misma manera, el diseño de prácticas experimentales cuyo enfoque central es la fotosíntesis (González et al., 2014; Macías, 2013; Sáenz, 2012) da cuenta de una exigencia silenciosa para transformar la manera de abordar este concepto desde el aula. Igualmente, nos encontramos con tareas investigativas que abarcan los aspectos básicos de la fotosíntesis (Caballero, 2004; Pérez, 2009) y con aquellos que hacen referencia al esquema de relación de la fotosíntesis con la conservación de la energía (Díaz et al., 2013; Manrique, 2003). Es decir, la percepción de la fotosíntesis ancla un sinnúmero de categorías que han sido excluidas desde el diseño curricular. Entre tanto, en el estudio de Velásquez (2011) se identifican y se describen las ideas previas de los estudiantes para explicar y determinar los obstáculos más frecuentes en el aprendizaje del concepto.

Según lo anterior, este estudio busca reconocer las concepciones de los estudiantes de grado décimo y undécimo de la Institución Educativa San Miguel del Municipio de Coper, Boyacá, acerca de la fotosíntesis. En este sentido, nos planteamos la siguiente pregunta de investigación: ¿cuáles son las concepciones de los estudiantes de un contexto rural sobre la fotosíntesis?

\section{Metodología}

Este trabajo se desarrolla desde el enfoque cualitativo, que busca caracterizar las concepciones de los estudiantes de grado décimo y undécimo de un contexto rural acerca 
de la fotosíntesis. En este estudio, se han utilizado las respuestas de un cuestionario para recoger las ideas de los estudiantes, puesto que no se manifiestan abiertamente. Por esta razón, las investigadoras las infieren a través de la aplicación de un test inicial. En nuestro caso, utilizamos un cuestionario de 30 preguntas sobre el concepto. Este abordó aspectos como intercambio gaseoso, respiración y nutrición autótrofa.

Para la valoración de las respuestas, se caracterizaron las concepciones de los participantes por subcategorías que guardan alguna relación entre sí. En la siguiente tabla, se hace una relación de las preguntas planteadas $-1,3,5,7,8,11,15,17,19$ y 27 - que responden a la categoría que permite abordar las ideas de los estudiantes acerca de la fotosíntesis, junto con sus respectivos criterios de categorización.

\section{Tabla 1}

Criterios de categorización con respecto a las concepciones de los estudiantes acerca de la importancia de la fotosintesis

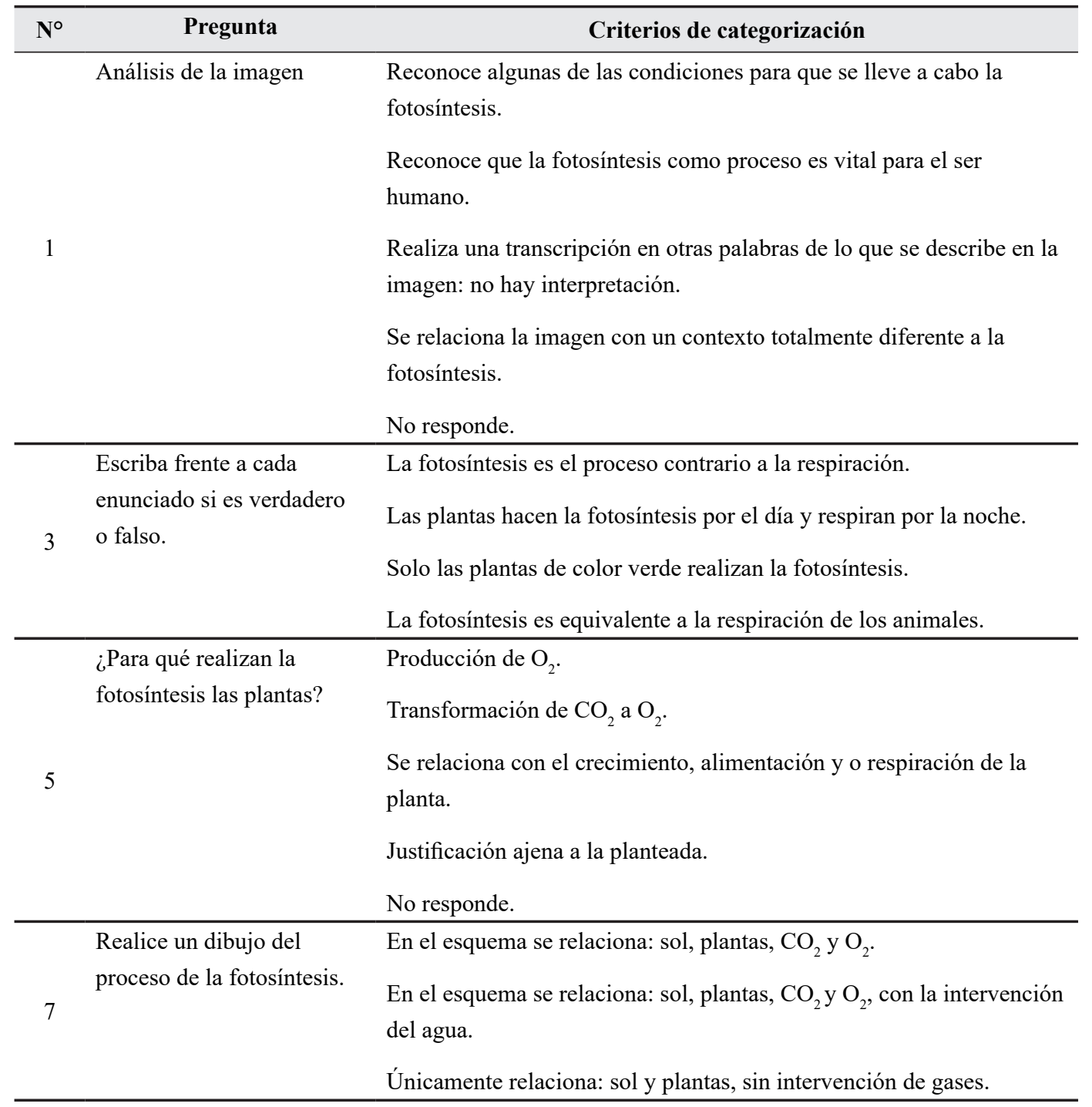




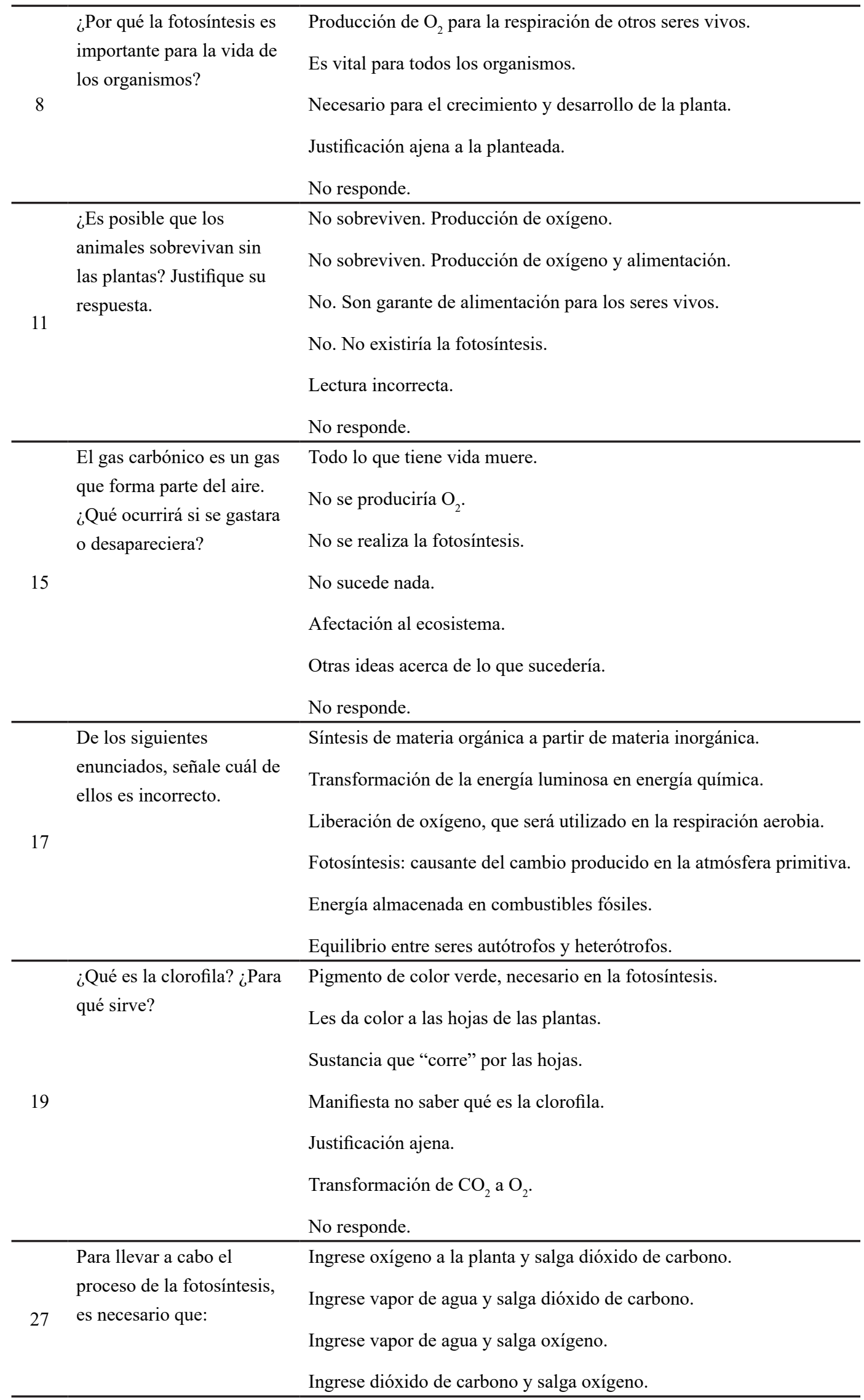




\section{Participantes}

En el cuestionario participaron 24 estudiantes, con edades comprendidas entre los $14 \mathrm{y}$ 18 años, que cursan en 2020, en la Institución Educativa San Miguel, el grado $10^{\circ}-58.4 \%$ y $11^{\circ}-41,6 \%$ - El $67 \%$ son mujeres. La muestra corresponde a la totalidad de estudiantes que cursan la educación media en la institución de carácter oficial y de contexto rural.

A los participantes se les informó sobre la finalidad del estudio. Mediante consentimiento informado se solicitó su participación en la investigación. Ellos de manera voluntaria manifestaron su interés. El cuestionario (anexo A) fue resuelto en el primer periodo académico de 2020.

\section{Contexto institucional}

En la actualidad, la Institución Educativa San Miguel tiene cinco sedes de primaria y una de secundaria. Está ubicada en la vereda Turtur, sector San Miguel del municipio de Coper, situado en la Provincia de Occidente. Se encuentra aproximadamente a $65 \mathrm{~km}$ de Chiquinquirá y $133 \mathrm{~km}$ de Tunja, capital del departamento de Boyacá. Es la única del sector y lleva 11 años de servicio. Atiende aproximadamente a 128 estudiantes activos, distribuidos en los diferentes niveles de escolaridad: básica primaria $-45 \%$-, básica secundaria $-36 \%-$ y educación media $-19 \%-$, por lo cual se trata de un muestreo no probabilístico (Hernández et al., 2010). Los estudiantes provienen de los diferentes sectores de la vereda. El nivel socioeconómico de las familias es bajo, debido a que viven del cultivo de café, caña de azúcar y ganadería en pequeña escala. En su gran mayoría son jornaleros.

\section{El instrumento}

Para conocer las concepciones de los estudiantes, se adaptó un cuestionario de 30 preguntas que se relacionan con la fotosíntesis y otros conceptos - respiración, nutrición autótrofa e intercambio gaseoso-; algunas de ellas son preguntas abiertas $-50 \%$-, de selección múltiple $-30 \%$-, de relación $-10 \%$-, representación gráfica $-6,7 \%-$ y de verdadero-falso $-3,3 \%$ - El instrumento empleado ha sido tomado y adaptado de otros trabajos investigativos (Angosto, 2018; Cañal, 1990; Macías, 2013) que se relacionan con el concepto de estudio.

A partir de la agrupación de las preguntas, estos conceptos asociados dan lugar a las categorías de análisis (tabla 2) que se van a emplear con el fin de conocer las concepciones de los estudiantes. En tal sentido, se aborda el estudio desde las perspectivas de los participantes, pues es necesario un análisis de los temas con los cuales los estudiantes relacionan el concepto y que a menudo se presta para confusiones (Angosto, 2018).

Tabla 2

Categorías de análisis

\begin{tabular}{lll}
\hline Categoría & Preguntas $\mathrm{N}^{\circ}$ & Propósito \\
\hline \multirow{2}{*}{$\begin{array}{l}\text { Nutrición } \\
\text { autótrofa }\end{array}$} & $\begin{array}{l}\text { Conocer las ideas previas de los estudiantes acerca del } \\
\text { mecanismo de transformación de sustancias inorgánicas }\end{array}$ \\
\hline
\end{tabular}




\begin{tabular}{lll}
\hline $\begin{array}{l}\text { Concepto de } \\
\text { respiración y } \\
\text { características }\end{array}$ & $2,12,18,23,25$ y 30 & $\begin{array}{l}\text { Comprender cómo los estudiantes relacionan el concepto } \\
\text { de la respiración y si lo reconocen como un proceso } \\
\text { propio de todos los seres vivos, incluidas las plantas junto } \\
\text { con sus características fundamentales. }\end{array}$ \\
\hline $\begin{array}{l}\text { Importancia de la } \\
\text { fotosíntesis }\end{array}$ & $\begin{array}{l}1,3,5,7,8,11,15,17, \\
1927\end{array}$ & $\begin{array}{l}\text { Analizar las nociones que se tienen respecto a la } \\
\text { importancia de la fotosíntesis en un determinado } \\
\text { ecosistema. }\end{array}$ \\
\hline $\begin{array}{l}\text { Intercambio } \\
\text { gaseoso }\end{array}$ & 24,26 y 28 & $\begin{array}{l}\text { Verificar si los estudiantes identifican las estructuras que } \\
\text { participan en el proceso de intercambio gaseoso que se } \\
\text { presenta en las plantas y cómo se lleva a cabo. }\end{array}$ \\
\hline
\end{tabular}

\section{Resultados y discusión}

Los resultados se fundamenta en las concepciones que los estudiantes de la educación media de un contexto rural proporcionan sobre el concepto de la fotosíntesis a través del análisis individual al instrumento empleado.

\section{Importancia de la fotosíntesis}

En este artículo solo presentaremos los resultados obtenidos a los cuestionamientos 1, $3,5,7,8,11,15,17,19$ y 27 , dado que responden al concepto de la fotosíntesis, base de este estudio.

\section{Importancia de la fotosíntesis: descripción gráfica}

En la tabla 3, se presentan las respuestas de una pregunta que desde una imagen indaga sobre la importancia de la fotosíntesis. Se destaca que un $33.3 \%$ de los participantes reconocen algunas de las condiciones para que las plantas lleven a cabo este proceso. Incluso, uno de ellos se atreve a presentar una analogía de lo que logra interpretar:

- E7: hasta que no realice la fotosíntesis, no se puede ir de la mesa porque necesita alimentarse y necesita nutrientes. Es como cuando mi mamá regaña a mi sobrino por no comer. En esta imagen se ve como si muy pronto se fuera a oscurecer y pues ya no puede realizar su fotosíntesis porque necesita del sol.

Respuestas como la siguiente sustentan la base para que el proceso de fotosíntesis se lleve a cabo:

- E9: que no se puede ir hasta que reciba sol y agua para vivir.

A su vez, se evidencia que la consideran como un proceso fundamental que deriva en lo siguiente:

- E10: pues que expulse el $\mathrm{O}_{2}$ y reciba el $\mathrm{CO}_{2}$ proveniente como producto final de la respiración de otros organismos.

Sin embargo, también se encuentran concepciones ajenas a lo estipulado en la pregunta, donde un $8.3 \%$ muestra una interpretación errada de la misma. Por su parte, un $33.3 \%$ transcribe lo expuesto en la imagen, sin llegar a ninguna exégesis al respecto.

Por consiguiente, los participantes presentan dificultades para una comprensión básica acerca de la fotosíntesis y de cómo un ecosistema funciona a través de esta (Skribe \& Strgar, 2017). Sin embargo, en este punto se hace énfasis en su importancia como proceso elemental 
en el planeta Tierra, ya que la existencia de otros seres vivos depende fundamentalmente de la fotosíntesis, pero los estudiantes no dan cuenta de esta distinción.

Tabla 3

Porcentaje de respuestas en la pregunta 1

\begin{tabular}{ccccc}
\hline \multicolumn{5}{c}{ Explicación de la imagen } \\
\hline $\begin{array}{c}\text { Reconoce } \\
\text { algunas de las } \\
\text { condiciones } \\
\text { para que se } \\
\text { lleve a cabo la } \\
\text { fotosíntesis. }\end{array}$ & $\begin{array}{c}\text { Reconoce que la } \\
\text { fotosíntesis como }\end{array}$ & $\begin{array}{c}\text { peceso es vital para } \\
\text { transcripción en } \\
\text { otras palabras de lo } \\
\text { que se describe en } \\
\text { la imagen: no hay } \\
\text { interpretación. }\end{array}$ & $\begin{array}{c}\text { Se relaciona la } \\
\text { imagen con un } \\
\text { conto totalmente } \\
\text { diferente a la } \\
\text { fotosíntesis. }\end{array}$ & No responde. \\
\hline 8 & 2 & 8 & 2 & 4 \\
\hline $\mathbf{3 3 . 3} \%$ & $\mathbf{8 . 3 \%}$ & $\mathbf{3 3 . 3} \%$ & $\mathbf{8 . 3 \%}$ & $\mathbf{1 6 . 6 \%}$ \\
\hline
\end{tabular}

\section{Fotosíntesis vs. Respiración}

En la tabla 4, se muestran aquellas preguntas que presentan dos opciones de respuesta verdadera o falsa-. Algunos de estos enunciados son formulados de manera que se puedan establecer patrones de comparación con otras cuestiones que se asimilan en su contenido, así que conocer la respuesta a uno de estos enunciados hace posible inferir la respuesta de la otra. Un 50 \% de los participantes elige la falsedad de que la fotosíntesis se realiza por el día y la respiración, en cambio, por la noche. Sabemos que la fotosíntesis y la respiración no tienen las mismas funciones y que es a través de la fotosíntesis que las plantas forman los compuestos orgánicos que son necesarios para mantener su homeostasis.

Tabla 4

Porcentaje de respuestas en la pregunta 3

\begin{tabular}{lccc}
\hline \multicolumn{4}{c}{ Escriba frente a cada enunciado si es verdadero o falso } \\
\hline \multicolumn{1}{c}{ Subcategorías } & Verdadero & Falso & No responde \\
\hline La fotosíntesis es el proceso contrario a la respiración. & 14 & 9 & 1 \\
Las plantas hacen la fotosíntesis por el día y respiran por la noche. & 11 & 12 & 1 \\
Solo las plantas de color verde realizan la fotosíntesis. & 3 & 20 & 1 \\
La fotosíntesis es equivalente a la respiración de los animales. & 11 & 11 & 2 \\
\hline
\end{tabular}

La respiración celular tiene como propósito la obtención de la energía para que un organismo pueda llevar a cabo sus funciones vitales (Angosto, 2018). De esta manera, si un ser vivo está realizando uno de estos procesos, por ningún motivo el otro se detiene. Lo que sí es pertinente señalar es que el proceso fotosintético se presenta en el día, dados los requerimientos de la luz solar. La respiración es un proceso continuo. En este sentido, es recurrente encontrar concepciones erróneas en los estudiantes, puesto que consideran que, mientras se produce uno de estos procesos, el otro se paraliza, o, sin duda, los toman como procesos contrarios. Incluso, toman como punto de partida que la fotosíntesis es un tipo de respiración propio de las plantas $-45,8 \%$-. Esto es un serio problema, ya que a partir 
de este tipo de respuestas es sencillo comprender por qué a los estudiantes se les dificulta manejar un lenguaje científico.

\section{¿Para qué la fotosíntesis?}

Se pueden encontrar respuestas un tanto conflictivas en donde no son claras las nociones de los estudiantes al respecto, pues, tal como aparece en la tabla 5 , el porcentaje de respuestas es altamente variado, por cuanto no hay relación alguna con la producción de oxígeno y carbohidratos como la glucosa de forma alterna. En algunos casos se describe por separado, sin recurrir a la mención de la glucosa. Por ejemplo, acá se afirma que la fotosíntesis es utilizada por las plantas:

- E19: para convertir dióxido en carbono a oxígeno que es lo que respiramos. Las plantas hacen trasformaciones bioquímicas con nutrientes del medio.

A pesar de que no profundiza en la idea de "transformaciones bioquímicas", se observa un intento por explicarla sin una relación estrecha con este último concepto.

\section{Tabla 5}

Porcentaje de respuestas en la pregunta 5

\begin{tabular}{ccccc}
\hline \multicolumn{5}{c}{ ¿Para qué realizan la fotosíntesis las plantas? } \\
\hline Producción de $\mathrm{O}_{2}$ & $\begin{array}{c}\text { Transformación de } \\
\mathrm{CO}_{2} \text { a }_{2} .\end{array}$ & $\begin{array}{c}\text { Se relaciona con el } \\
\text { crecimiento, alimentación } \\
\text { y o respiración de la } \\
\text { planta. }\end{array}$ & $\begin{array}{c}\text { Justificación } \\
\text { ajena a la } \\
\text { planteada. }\end{array}$ & No responde. \\
\hline 13 & 2 & 6 & 2 & 1 \\
\hline $\mathbf{5 4 , 2 \%}$ & $\mathbf{8 . 3 \%}$ & $\mathbf{2 5 \%}$ & $\mathbf{8 . 3 \%}$ & $\mathbf{4 , 2 \%}$ \\
\hline
\end{tabular}

Pérez (2009) incorpora elementos clave que fortalecen la formulación de los preconceptos necesarios para el ejercicio del aprendizaje de la fotosíntesis, ya que la trata como un proceso fundamental para la vida sobre la tierra. "El conocimiento básico de este proceso es esencial para entender las relaciones entre los seres vivos y la atmósfera, así como el balance de la vida sobre la tierra" (p. 1). Es importante resaltar que el entendimiento de la fotosíntesis como proceso vital ha de hacerse desde su origen. Con esto, favoreceríamos la construcción de significados desde el fortalecimiento de los ítems temáticos esenciales para su comprensión y se evitaría la formación de errores base.

\section{¿Cómo los estudiantes representan la fotosíntesis?}

Desde otra perspectiva, los esquemas juegan un papel interesante a la hora de representar con gráficas lo que las palabras no alcanzan (tabla 6). De esta manera, se encuentra que un 58,3 \% muestra como requisito esencial al sol, pero no se evidencia una posible transformación de energía luminosa a energía química. En este aspecto, los estudiantes no discriminan una fase dependiente de luz - claramente fotosintética, en la que la planta utiliza la energía lumínica como fuente principal para la producción de moléculas de ATPde otra estrictamente independiente de luz y metabólica en la que no existe participación alguna de fuentes externas de energía, sino que emplea ese ATP almacenado para sintetizar moléculas orgánicas a partir de moléculas inorgánicas (Angosto, 2018). Los estudiantes difícilmente hablan de una fase lumínica y de una fase oscura en el proceso de la fotosíntesis. 
Por lo tanto, no asimilan que "el oxígeno necesario para la respiración celular se obtiene a partir del proceso denominado respiración tanto en plantas como en animales" (Caño, 2019, p. 91). Lo representan de una manera gráfica como un simple intercambio gaseoso entre estos dos compuestos.

Tabla 6

Porcentaje de respuestas en la pregunta 7

\begin{tabular}{ccc}
\hline \multicolumn{3}{c}{ Realice un dibujo del proceso de la fotosíntesis } \\
\hline $\begin{array}{c}\text { En el esquema se muestra: sol, } \\
\text { plantas, } \mathrm{CO}_{2} \text { y } \mathrm{O}_{2} .\end{array}$ & $\begin{array}{c}\text { En el esquema se muestra: } \\
\text { sol, plantas, } \mathrm{CO}_{2} \text { y } \mathrm{O}_{2} \text {, con la } \\
\text { intervención del agua. }\end{array}$ & $\begin{array}{c}\text { Únicamente relaciona: sol y } \\
\text { plantas, sin intervención de gases. }\end{array}$ \\
\hline 9 & 1 & 14 \\
\hline $\mathbf{3 7 , 5} \%$ & $\mathbf{4 , 2} \%$ & $\mathbf{5 8 , 3} \%$ \\
\hline
\end{tabular}

\section{Importancia de la fotosíntesis para los seres vivos}

Frente la importancia de la fotosíntesis (tabla 7), se considera que uno de los productos finales y más importantes de todo este proceso es la producción de oxígeno, equivalente y necesario para la respiración de otros seres vivos, incluido el ser humano $-54,2 \%-$.

- E6: gracias a la fotosíntesis nos brinda el oxígeno para poder respirar.

Afirmaciones como esta aciertan en una de las finalidades del proceso. Sin embargo, los estudiantes no cuentan como resultado la producción de carbohidratos como la glucosa. Seguramente, no hay claridad en que las plantas llevan a cabo la fotosíntesis al aprovechar la luz del sol, la presencia de agua y el dióxido de carbono en su medio (Macías, 2013).

Por otro lado, se encuentran nociones que traslapan su importancia y las ideas alternativas no se hacen esperar, como en el siguiente caso:

- E24: porque se necesita para que se reproduzcan.

Sin duda, existe un desconcierto en el uso del concepto. Al respecto, Charrier et al. (2006) advierten la aparición de errores conceptuales, que corroboran la ausencia de la comprensión de los términos asociados a estos procesos —clorofila, hojas, oxígeno, dióxido de carbono, entre otros-. Cada pregunta dirigida a los estudiantes muestra resultados interesantes que se encaminan y coinciden con el trabajo de Cañal (1990), ya que se les dificulta señalar "el papel de la fotosíntesis como mecanismos de fabricación de alimentos para las plantas" (p. 285).

Tabla 7

Porcentaje de respuestas en la pregunta 8

\begin{tabular}{ccccc}
\hline \multicolumn{5}{c}{ ¿Por qué la fotosíntesis es importante para la vida de los organismos? } \\
\hline $\begin{array}{c}\text { Producción de } \mathrm{O}_{2} \\
\text { para la respiración }\end{array}$ & $\begin{array}{c}\text { Es vital para } \\
\text { todos los } \\
\text { de otros seres vivos. }\end{array}$ & $\begin{array}{c}\text { Necesario para el } \\
\text { crecimiento y desarrollo } \\
\text { organismos. }\end{array}$ & $\begin{array}{c}\text { Justificación ajena } \\
\text { de la planta. }\end{array}$ & No responde. \\
\hline 13 & 2 & 6 & 2 & 1 \\
\hline $\mathbf{5 4 , 2} \%$ & $\mathbf{8 . 3} \%$ & $\mathbf{2 5 \%}$ & $\mathbf{8 . 3} \%$ & $\mathbf{4 , 2} \%$ \\
\hline
\end{tabular}




\section{¿Sobrevivir sin las plantas?}

Ante la pregunta: ¿es posible que los animales sobrevivan sin las plantas? (tabla 8), ocurre lo mismo que en la descripción anterior. Se hace evidente que los conocimientos adquiridos en su etapa escolar solo les brindan una certeza asociada a la satisfacción de oxígeno para suplir las necesidades de otros. Notoriamente, los hallazgos encontrados en cada una de las respuestas conducen a hallar similitudes en su desarrollo, de allí que se incida nuevamente en un vacío de contenido. Como respuesta, se esperaría que el estudiante incluyera aspectos como la producción de oxígeno y la importancia de reconocer la fotosíntesis como aquel proceso que juega un papel preliminar para la respiración celular (Messig \& Groß, 2018).

\section{Tabla 8}

Porcentaje de respuestas en la pregunta 11

\begin{tabular}{lcc}
\hline \multicolumn{2}{c}{ ¿Es posible que los animales sobrevivan sin las plantas? Justifique su respuesta } \\
\hline \multicolumn{1}{c}{ Subcategorías } & Frecuencia & Porcentaje \\
\hline No sobreviven. Producción de oxígeno. & 8 & $33.3 \%$ \\
No sobreviven. Producción de oxígeno y alimentación. & 5 & $20,8 \%$ \\
No. Son fuente de alimentación para los seres vivos. & 7 & $29,2 \%$ \\
No. No existiría la fotosíntesis. & 1 & $4,2 \%$ \\
Lectura incorrecta. & 2 & $8.3 \%$ \\
No responde. & 1 & $4,2 \%$ \\
\hline
\end{tabular}

Cañal (1990) señala que la "nutrición de las plantas verdes [...] suele estar presente también en las aulas de primaria, y es allí donde se generarán algunas de las ideas que tendrán una mayor incidencia en la construcción escolar relativa a este campo conceptual" (p. 98). De esta manera, lo presentado en la tabla 9 muestra que los participantes tienen dificultades en el uso de la terminología para referirse al proceso metabólico (Velásquez, 2011). Un 29,2 \% estima que la desaparición del gas carbónico lleva a la afectación directa de los seres vivos, ya que su posibilidad de vida se apagaría, tal como se menciona a continuación

- E1: se morirían las plantas, los animales, las personas.

Como complemento a su respuesta, otro estudiante afirma:

- E2: no habría oxígeno.

Este sería el argumento por el cual los seres vivos desaparecerían junto con el dióxido de carbono de un ecosistema.

\section{Tabla 9}

Porcentaje de respuestas en la pregunta 15

\begin{tabular}{lcc}
\hline \multicolumn{2}{c}{ El gas carbónico es un gas que forma parte del aire. ¿Qué ocurriría si se gastara o desapareciera? } \\
\hline \multicolumn{1}{c}{ Subcategorías } & Frecuencia & Porcentaje \\
\hline Todo lo que tiene vida muere. & 7 & $29,2 \%$ \\
No se produciría $\mathrm{O}_{2}$ & 6 & $25 \%$ \\
No se realiza la fotosíntesis. & 4 & $16.6 \%$ \\
No sucede nada. & 1 & $4,2 \%$
\end{tabular}




\begin{tabular}{lcc} 
Afectación al ecosistema. & 1 & $4,2 \%$ \\
Otras ideas acerca de lo que sucedería. & 4 & $16.6 \%$ \\
No responde. & 1 & $4,2 \%$ \\
\hline
\end{tabular}

\section{¿Solo producción de oxígeno?}

Al observar los resultados de la tabla 10, tenemos que el 33,3\% de los estudiantes, tanto de décimo como de undécimo, piensa que de la fotosíntesis no depende la energía almacenada en combustibles y continúan afirmando que su finalidad principal y mayoritaria es la producción de oxígeno. En trabajos anteriores (Charrier et al., 2006) se encuentran resultados afines al punto de vista de los estudiantes y que permanece a medida que avanzan en sus niveles de escolaridad. Así, el estudiante concibe la idea de que las plantas mediante la fotosíntesis producen oxígeno para que otros seres vivos puedan respirar. Les es difícil comprender que el oxígeno simplemente es un subproducto que tiene como propósito sintetizar materia orgánica que luego es aprovechada por la misma planta como alimento (Sáenz, 2012). Aquí es clave mencionar que el aporte suministrado desde el análisis de cada una de las concepciones obtenidas por los participantes da lugar a encontrar una "existencia de mecanismos generales de vaciamiento discursivo en el aula. Entendemos por vaciamiento discursivo, la desnaturalización de la función del lenguaje como sustento de los contenidos disciplinares específicos" (Galagovsky et al., 1998, p. 315).

Tabla 10

Porcentaje de respuestas en la pregunta 17

\begin{tabular}{lcc}
\hline \multicolumn{1}{c}{ De los siguientes enunciados, señale cuál de ellos es incorrecto } \\
\hline \multicolumn{1}{c}{ Subcategorías } & Es incorrecto & No responde \\
\hline Síntesis de materia orgánica a partir de materia inorgánica. & 5 & 3 \\
Transformación de la energía luminosa en energía química. & 2 & 3 \\
Liberación de oxígeno, que será utilizado en la respiración aerobia. & 7 & 3 \\
Fotosíntesis: causante del cambio producido en la atmósfera primitiva. & 7 & 3 \\
Energía almacenada en combustibles fósiles. & 8 & 3 \\
Equilibrio entre seres autótrofos y heterótrofos. & 4 & 3 \\
\hline
\end{tabular}

¿Qué es la clorofila?

$\mathrm{Al}$ analizar las respuestas obtenidas a la pregunta 19 , se constata que los estudiantes no tienen clara la funcionalidad de la clorofila. No la reconocen como aquel pigmento que se relaciona de forma directa con la conversión de energía luminosa en energía química (Sáenz, 2012). Simplemente la describen como la responsable de dar el color característico de las plantas - $50 \%$ - Sin embargo, un $16,6 \%$ se atreve a mencionar que es necesaria en la fotosíntesis. Esto se evidencia a continuación:

- E7: es un componente que utiliza la luz para llenar de energía las plantas y les da un color verde en el proceso de la fotosíntesis.

- E22: la clorofila es una sustancia que tienen las plantas en sus hojas y ayuda en el proceso de la fotosíntesis.

Aquí se hace evidente un esquema básico en la conceptualización del término que para 
los participantes no encaja armónicamente en el proceso de la fotosíntesis.

\section{Tabla 11}

Porcentaje de respuestas en la pregunta 19

\begin{tabular}{lcc}
\hline \multicolumn{3}{c}{ ¿Qué es la clorofila? ¿Para qué sirve? } \\
\hline \multicolumn{1}{c}{ Subcategorías } & Frecuencia & Porcentaje \\
\hline Pigmento de color verde, necesario en la fotosíntesis & 4 & $16.6 \%$ \\
Les da color a las hojas de las plantas. & 12 & $50 \%$ \\
Sustancia que "corre" por las hojas. & 3 & $12,5 \%$ \\
Manifiesta no saber qué es la clorofila. & 1 & $4,2 \%$ \\
Justificación ajena. & 2 & $8.3 \%$ \\
Transformación de $\mathrm{CO}_{2}$ a $\mathrm{O}_{2}$. & 1 & $4,2 \%$ \\
No responde. & 1 & $4,2 \%$ \\
\hline
\end{tabular}

¿Qué necesita la planta para realizar la fotosíntesis?

Para la pregunta 27, se encontró que un 83,3 \% de los estudiantes afirma que, para que la fotosíntesis se lleve a cabo, se requiere el ingreso de dióxido de carbono y la salida de oxígeno. Esto contrasta con las ideas encontradas que dinamizan el proceso con un simple intercambio gaseoso. Lo anterior puede considerarse un error de base ampliamente significativo, porque ilustra un desconocimiento del proceso en sí mismo (Angosto, 2018). Es necesario resaltar que, pese a que los estudiantes han acertado en su mayoría a este cuestionamiento, difícilmente asumen la concepción de que los alimentos también pueden ser de carácter gaseoso, como es el caso del dióxido de carbono. Consideran que la planta solamente absorbe sustancias líquidas o sólidas. Igualmente, a partir de las nociones encontradas en los participantes, se puede afirmar que la absorción de este compuesto gaseoso está relacionada estrechamente con la respiración en lugar de la fotosíntesis (Messig \& Groß, 2018).

Tabla 12

Porcentaje de respuestas en la pregunta 27

\begin{tabular}{cccc}
\hline \multicolumn{4}{c}{ Para llevar a cabo el proceso de la fotosíntesis, es necesario que: } \\
\hline $\begin{array}{c}\text { Ingrese oxígeno a la } \\
\text { planta y salga dióxido de } \\
\text { carbono. }\end{array}$ & $\begin{array}{c}\text { Ingrese vapor de agua y } \\
\text { salga dióxido de carbono. }\end{array}$ & $\begin{array}{c}\text { Ingrese vapor de agua y } \\
\text { salga oxígeno. }\end{array}$ & $\begin{array}{c}\text { Ingrese dióxido de } \\
\text { carbono y salga } \\
\text { oxígeno. }\end{array}$ \\
\hline 2 & 1 & 1 & 20 \\
\hline $\mathbf{8 . 3} \%$ & $\mathbf{4 , 2} \%$ & $\mathbf{4 , 2} \%$ & $\mathbf{8 3 , 3} \%$ \\
\hline
\end{tabular}

Finalmente, indicamos que son innumerables los estudios enfocados a indagar las concepciones que tienen los estudiantes respecto a la fotosíntesis. Se encuentran en ellos ideas alternativas y confusiones conceptuales de base. Difícilmente los estudiantes llegan a comprender el concepto de manera global e integrado, pues en las nociones analizadas hay contradicciones y vacíos de información que dificultan la comprensión y, en consecuencia, su aprendizaje. En todo esto, el "actor fundamental para lograr los procesos de calidad es el [...] docente" (Serrato, 2018, p. 181), a quien también se debe considerar desde su formación como sujeto social (Torres et al., 2014). 


\section{Consideraciones finales}

En cuanto a las ideas de los participantes, se destaca, acerca de los conocimientos previos de la fotosíntesis, que dan cuenta de la disipación y falta de organización al momento de plasmar sus ideas al respecto. A su vez, emplean con gran frecuencia términos que carecen de significado real para quien escribe - energía, transformación química, intercambio gaseoso, estomas, alimento, nutrición, entre otros-. En la mayoría de las situaciones encontradas, los estudiantes acuden a recuerdos ambiguos de las explicaciones brindadas durante el trascurso de su vida escolar.

En particular, las explicaciones brindadas por los docentes en años anteriores se han abandonado, quizá porque para ellos no contienen relevancia en su significado y no fueron fácilmente asimilables a través de situaciones didácticas que permitieran una mayor apropiación entre lo que tienen como concepto previo y aquello que hace parte de nuevas experiencias. Sin embargo, el conocimiento de estos conceptos erróneos hace que sea fundamental revisar los procesos de educabilidad que se llevan a cabo en la educación inicial. Desde esta perspectiva, adquiere sentido asumir el entorno natural en el que se encuentran implícitos los estudiantes para la enseñanza de las ciencias.

En particular, los estudiantes confunden la fotosíntesis como una forma de respiración de las plantas. Creen que la planta produce oxígeno exclusivamente para satisfacer el requerimiento de otros seres vivos, excepto el propio. Consideran el agua como compuesto esencial para la fotosíntesis, al igual que el sol, pero difícilmente dan el valor energético que la luz solar representa para la planta en sus mecanismos de trasformación de energía lumínica a química, lo que para los demás seres vivos es uno de sus productos finales, la glucosa. Dichas abstracciones son comprensibles, dado que se aprenden desde una descontextualización de las ciencias.

Es de esperarse que los estudiantes presenten un conocimiento científico fragmentado, en el que cohabitan ideas anticipadas producto de los niveles de escolaridad anteriormente cursados. La mayoría de los estudiantes participantes en este estudio mostró dificultades para representar que las plantas también respiran e incorporan $\mathrm{O}_{2}$ a su metabolismo. Otro aspecto fundamental fue que tampoco son ubicadas en la base de todas las cadenas tróficas, pero sí resaltan la idea de que son los mayores productores de oxígeno.

Este estudio tuvo como propósito reconocer las concepciones acerca de la fotosíntesis que presentan los estudiantes de grado décimo y undécimo de una institución educativa de contexto rural. Fue posible identificar los conceptos más comunes que a menudo conducen a ideas alternativas. Esta comprensión permitirá principalmente el desarrollo de una secuencia didáctica que hará énfasis en el desarrollo de la capacidad de comunicación y del interés por el concepto, desde el conocimiento del entorno, para evitar la formación de errores base, a partir de una ruta de actividades que guiarán a los estudiantes a emplear un lenguaje con contenido científico.

Lo anterior evidencia la necesidad de profundizar en esos procesos de discurso en el aula para ampliar las nociones específicas de los estudiantes a través de procesos de enseñanza y que resulten suficientes para superar las dificultades y deficiencias en el manejo de preconceptos, a través de una continuidad en la que se enriquezca el lenguaje para reconstruir modelos científicos. 


\section{Referencias}

Amiguinho, A. (2011). La escuela en el medio rural: educación y desarrollo local. Profesorado. Revista de currículum y formación del profesorado, 15(2), 25-37.

Angosto, I. (2018). Didáctica de la nutrición vegetal. Análisis de los principales manuales de fisiología vegetal y de las concepciones de los estudiantes del Máster Universitario en Formación del Profesorado de ESO y Bachillerato de la UCM [Tesis doctoral, Universidad Complutense de Madrid, España]. https://dialnet.unirioja.es/servlet/ tesis? codigo $=133992$

Astudillo, H., \& Gene, A. (1984). Errores conceptuales en biología. La fotosíntesis de las plantas verdes. Enseñanza de las Ciencias, 2(1), 15-16.

Barrutia, O., Ruiz, A., Zuazagoitia, D., Taldea, S., \& Díez, J. (2016). ¿Ha mejorado el conocimiento sobre la nutrición de las plantas desde los años 90 ? Un análisis temporal con alumnado de primaria y secundaria. Campo Abierto, 35(1), 217-231.

Bustos, A. (2011). Investigación y escuela rural: ¿irreconciliables? Profesorado. Revista de currículum y formación del profesorado, 15(2), 155-170.

Caballero, P. (2004). Manual teórico de Fotosíntesis, ciclo de Calvin y destinos del carbono fotoasimilado. Universidad de las Palmas de Gran Canaria.

Cañal, P. (1990). La enseñanza en el campo conceptual de la nutrición de las plantas verdes: un estudio didáctico en la educación básica [Tesis doctoral, Universidad de Sevilla, España]. https://dialnet.unirioja.es/servlet/tesis? codigo $=22796$

Cañal, P. (1991). Las concepciones de los alumnos y alumnas sobre la nutrición de las plantas verdes. Investigación en la Escuela, (13), 97-113.

Caño, L. (2019). Concepciones sobre nutrición vegetal y relaciones tróficas en función del bagaje educativo: implicaciones para el futuro profesorado. Didáctica de las Ciencias Experimentales $y$ Sociales, (37), 89-106. https://doi.org/10.7203/dces.37.13285

Charrier, M., Cañal, P., \& Rodrigo, M. (2006). Las concepciones de los estudiantes sobre la fotosíntesis y la respiración: una revisión sobre la investigación didáctica en el campo de la enseñanza y el aprendizaje de la nutrición de las plantas. Enseñanza de las ciencias, 24(3), 401-410.

Díaz, M., Guzmán, E., Rodiño Y., \& Aguilar, Y. (2012). La formalización del concepto de fotosíntesis a partir del principio de conservación de la energía en la enseñanza de las ciencias. Un análisis histórico-epistemológico desde la perspectiva de Mayer. Revista EDUCyT, (13), 52-67.

Galagovsky, L., Bonán, L., \& Adúriz, A. (1998). Problemas con el lenguaje científico en la escuela. Un análisis desde la observación de clases de Ciencias Naturales. Enseñanza de las Ciencias, 16(2), 315-321.

Garnica, S., \& Roa, R. (2013). Conocimiento didáctico del contenido sobre fotosíntesis de dos profesores de los grados sexto y noveno de educación básica secundaria de un colegio privado en Bogotá-Colombia. Biografía: Escritos sobre la Biología y su Enseñanza, 5(8), 50-76. https://doi.10.17227/20271034.8biografia50.76 
Gómez, G. (2014). Propuesta didáctica para la enseñanza de la fotosíntesis dirigida a estudiantes del ciclo V del Colegio Rural Pasquilla I. E. D. [Tesis de maestría, Universidad Nacional, Bogotá, Colombia]. Repositorio institucional. https://repositorio.unal.edu.co/ handle/unal/52133

Gómez, D., \& Velazco, D. (2015). Ideas previas sobre el reino vegetal en niños entre los 7 y 10 años de tercero de primaria de la Escuela Normal Superior Distrital María Montessori. Biografía. Escritos sobre la Biología y su Enseñanza, Edición Extraordinaria, 1460-1470. https://doi.org/10.17227/20271034.vol.0num.0bio-grafia1460.1470

González, C., García, S., \& Martínez, C. (2003). ¿A qué contenidos relacionados con la fotosíntesis dan más importancia los textos escolares de secundaria? Enseñanza de las Ciencias, (Extra), 77-88.

González, C., Martínez, C., \& García, S. (2014). El modelo de nutrición vegetal a través de la historia y su importancia para la enseñanza. Revista Eureka sobre Enseñanza y Divulgación de las Ciencias, 11(1), 2-12. https://doi.10.25267/Rev_Eureka_ensen_ divulg_cienc.2014.v11.i1.02

Hernández, R., Fernández, C., \& Baptista, M. (2010). Metodología de la investigación. McGraw-Hill.

Herrera, L., \& Buitrago, R. (2015). Educación rural en Boyacá, fortalezas y debilidades desde la perspectiva del profesorado. Praxis \& Saber, 6(12), 169-190. https://doi. org/10.19053/22160159.3768

Keleş, E., \& Kefelib, P. (2010). Determination of student misconceptions in "photosynthesis and respiration" unit and correcting them with the help of cai material. Procedia Social and Behavioral Sciences, 2(2), 3111-3118. https://doi.org/10.1016/j.sbspro.2010.03.474

Lemke, J. (1997). Aprender a hablar ciencia: lenguaje, aprendizaje y valores. Ediciones Paidós Ibérica, S.A.

Macías, L. (2013). Diseño de prácticas experimentales de fotosíntesis para ciclo 3 [Tesis de maestría no publicada]. Universidad Nacional, Bogotá, Colombia.

Manrique, E. (2003). Los pigmentos fotosintéticos, algo más que la captación de luz. Ecosistemas, 12(1). https://www.revistaecosistemas.net/index.php/ecosistemas/article/ view/250

Messig, D., \& Groß, J. (2018). Understanding Plant Nutrition - The Genesis of Students' Conceptions and the Implications for Teaching Photosynthesis. Education Sciences, 132(8), 1-10. https://doi.org/10.3390/educsci8030132

Pérez, E. (2009). Fotosíntesis: aspectos básicos. Reduca Biología. Serie Fisiología Vegetal, 2(3), 1-47.

Sáenz, J. (2012). La fotosíntesis, concepciones, ideas alternativas y analogías. Unidad didáctica dirigida a estudiantes de los ciclos 3 y 4 de educación básica del colegio José María Carbonell [Tesis de maestría, Universidad Nacional, Bogotá, Colombia]. Repositorio institucional. https://repositorio.unal.edu.co/bitstream/handle/unal/10416/ jorgeenriquesaenzguarin.2012.pdf? sequence $=1$ \&isAllowed $=\mathrm{y}$ 
Sanmartí, N. (2007). Hablar, leer y escribir para aprender ciencia. En P. Fernández (Coord.), La competencia en comunicación lingüística en las áreas del currículo (pp. 1-21). MEC.

Serrato, D. (2018). El discurso del desarrollo y la educación en lo contemporáneo: ¿es pertinente formar en ciencias? Praxis \& Saber, 9(20), 167-189. https://doi. org/10.19053/22160159.v9.n20.2018.8303

Skribe, D., \& Strgar, J. (2017). Scientific conceptions of photosynthesis among primary school pupils and student teachers of biology. CEPS Journal, 7(1), 49-68. https://doi. org/10.26529/cepsj.14

Svandova, K. (2014). Secondary School Students' Misconceptions about Photosynthesis and Plant Respiration: Preliminary Results. Eurasia Journal of Mathematics, Science \& Technology Education, 10(1), 59-67.

Torres, N., \& Montenegro, C. (2018). ¿Cómo interpretan los niños prácticas experimentales relacionadas con el concepto de densidad? Revista Praxis \& Saber, 9(21), 21-45. https:// doi.org/10.19053/22160159.v9.n21.2018.6030

Torres, N., Pachón, N., Medina, N., \& Amézquita, J. (2014). Reflexiones críticas sobre la formación del sujeto social en un programa de formación superior. Praxis \& Saber, 5(9), 127-147. https://doi.org/10.19053/22160159.2997

Velásquez, L. (2011). Modelos explicativos sobre el concepto de nutrición en plantas en estudiantes de básica secundaria rural [Tesis de maestría, Universidad Nacional, Bogotá, Colombia]. Repositorio institucional. https://repositorio.unal.edu.co/bitstream/handle/ unal/9102/8410005.2012_.pdf? sequence $=1$ \&isAllowed $=y$ 
ANEXO A

UNIVERSIDAD PEDAGÓGICA Y TECNOLÓGICA DE COLOMBIA

MAESTRÍA EN EDUCACIÓN

Trabajo de grado: HABLAR CIENCIA DESDE LA EXPERIMENTACIÓN DEL ENTORNO NATURAL PARA EL APRENDIZAJE DE LA FOTOSÍNTESIS

Cuestionario diagnóstico para estudiantes de grado $10^{\circ}$ y $11^{\circ}$ de la I.E. San Miguel acerca de los conocimientos previos sobre la fotosíntesis

Estimado (a) estudiante, le invito para que conteste en los espacios correspondientes las preguntas relacionadas con el tema de la fotosíntesis y otros conceptos asociados. $\mathrm{Su}$ finalidad está en analizar cómo los estudiantes de un contexto rural hablan ciencia desde la experimentación del entorno natural para el aprendizaje del concepto. Con ello, se busca encontrar la relación de los preconceptos que usted tiene frente a la temática y así dar desarrollo a la propuesta de investigación titulada Hablar ciencia desde la experimentación del entorno natural para el aprendizaje de la fotosíntesis.

Lea detenidamente el enunciado de cada pregunta y conteste de acuerdo a los conceptos aprendidos a lo largo de su experiencia escolar.

1. Explique con sus propias palabras la siguiente imagen.

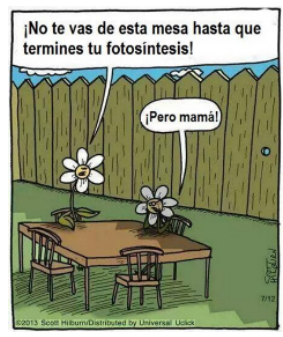

2. Señale con una $X$ los seres vivos que respiran.

$\begin{array}{ll}\text { Perro } & \text { Hierba } \\ \text { Gusano } & \text { Hongo } \\ \text { Musgo } & \text { Mejillón } \\ \text { Coral } & \text { Manzana } \\ \text { Algas } & \text { Pez }\end{array}$

3. Escriba frente a cada enunciado si es verdadero o falso.

a. La fotosíntesis es el proceso contrario a la respiración. ( )

b. Las plantas hacen la fotosíntesis por el día y respiran por la noche. ( )

c. Solo las plantas de color verde realizan la fotosíntesis. ( )

d. Los gases son sustancias. ( )

e. Es peligroso dormir con plantas por la noche porque nos podemos quedar sin oxígeno. ( )

f. El gas $\mathrm{CO}_{2}$ es alimento para las plantas. ( ) 
g. Las raíces son como la boca de las plantas. ( )

h. Las plantas comen tierra. ( )

i. La fotosíntesis es equivalente a la respiración de los animales. ( )

j. El alimento de las plantas son los restos de animales muertos y excrementos de animales (abono). ( )

4. De los siguientes compuestos señale cuáles son orgánicos y cuáles son inorgánicos.

$\begin{array}{ll}\mathrm{CO}_{2} \text { (Dióxido de carbono) } & \mathrm{C}_{6} \mathrm{H}_{12} \mathrm{O}_{6} \text { (Glucosa) } \\ \mathrm{O}_{2} \text { (Oxígeno) } & \text { Célula } \\ \mathrm{NaCl} \text { (Sal) } & \mathrm{H}_{2} \mathrm{O} \text { (Agua) } \\ \text { Clorofila } & \text { Cloroplastos }\end{array}$

5. ¿Para qué realizan la fotosíntesis las plantas?

6. ¿Qué es la nutrición autótrofa?

7. Realice un dibujo del proceso de la fotosíntesis.

8. ¿Por qué la fotosíntesis es importante para la vida de los organismos?

9. ¿Qué recursos permiten que las plantas se desarrollen y crezcan?

10. ¿Por qué las plantas son llamadas organismos productores?

11. ¿Es posible que los animales sobrevivan sin las plantas? Justifique su respuesta.

12. ¿Qué sustancia y/o compuesto del aire es la que toman las plantas como alimento y qué parte toman para respirar?

13. Si una planta pierde sus hojas, ¿qué le ocurrirá? Explique su respuesta.

a. ¿Podrá alimentarse?

b. ¿Podrá respirar? 
14. Imagine que cada hoja de una planta fuera como una fábrica.

a. ¿Qué hace esa fábrica?

b. ¿Qué sustancias necesita para fabricar sus productos?

c. ¿De dónde obtiene la energía para poder funcionar?

15. El gas carbónico es un gas que forma parte del aire. ¿Qué ocurrirá si se gastara o desapareciera?

16. Si unos gusanos se comen las raíces de una planta, ¿qué le pasará a la planta? ¿Por qué?

17. De los siguientes enunciados, señale cuál de ellos es incorrecto.

a. La síntesis de materia orgánica a partir de la materia inorgánica se realiza fundamentalmente mediante la fotosíntesis; luego irá pasando de unos seres vivos a otros mediante las cadenas tróficas, para ser transformada en materia propia por los diferentes seres vivos.

b. Produce la transformación de la energía luminosa en energía química, necesaria y utilizada por los seres vivos.

c. En la fotosíntesis se libera oxígeno, que será utilizado en la respiración aerobia como oxidante.

d. La fotosíntesis fue causante del cambio producido en la atmósfera primitiva, que era anaerobia y reductora.

e. De la fotosíntesis depende también la energía almacenada en combustibles fósiles como carbón, petróleo y gas natural.

f. El equilibrio necesario entre seres autótrofos y heterótrofos no sería posible sin la fotosíntesis.

18. Represente gráficamente la forma de respirar de una planta y de una persona. ¿En qué se parecen? ¿En qué se diferencian? 
19. ¿Qué es la clorofila? ¿Para qué sirve?

20. ¿Para qué cree que utiliza la planta la luz del sol?

21. ¿En las plantas, cómo llegan los nutrientes del suelo hasta las hojas? 


\section{PREGUNTAS DE SELECCIÓN MÚLTIPLE CON ÚNICA RESPUESTA}

Este tipo de preguntas consta de un enunciado y cuatro opciones de respuesta (a, b, c, d). Solo una de estas opciones responde correctamente la pregunta. Usted debe seleccionar la respuesta correcta y marcarla en su hoja.

Responda las preguntas 22 y 23 con base en el siguiente texto.

Los seres vivos requieren energía para realizar sus funciones vitales y la adquieren mediante la nutrición. Esta puede ser autótrofa como en las plantas, que realizan la fotosíntesis o heterótrofa, como en los demás organismos que deben conseguir de alguna manera su alimento.

22. La diferencia entre las plantas y los animales, en cuanto a la nutrición es que

a. las plantas son herbívoras y los animales carnívoros.

b. las plantas no comen y los animales comen mucho.

c. los animales buscan su alimento y las plantas fabrican el suyo.

d. los animales cazan su propio alimento y las plantas lo buscan.

23. Los seres vivos que no obtienen energía

a. pueden realizar sus funciones vitales.

b. pueden vivir sin sus funciones vitales.

c. pueden realizar sus funciones sin energía.

d. no pueden realizar sus funciones vitales.

24. El carbono necesario para la fotosíntesis se obtiene a través del $\mathrm{CO}_{2}$ que entra a las plantas gracias a los estomas. Cuando las hojas tienen abiertos los estomas entra $\mathrm{CO}_{2}$ pero se evapora agua. En los desiertos se observan plantas, como los cactus, que han encontrado una alternativa para minimizar el problema. De las siguientes estrategias la más adecuada para resolver este problema es

a. Mantener cerrados los estomas permanentemente.

b. Abrir los estomas solo durante los periodos de lluvia.

c. Abrir los estomas en la noche, cuando la temperatura es menor.

d. Mantener los estomas abiertos durante el día y cerrados en la noche.

25. Para obtener la energía a nivel celular se requiere de un gas, este es:

a. Dióxido de carbono

b. Oxígeno

c. Nitrógeno

d. Fosforo 
Responda las preguntas 26 a 28 con base en la siguiente información.

En las plantas, el intercambio gaseoso ocurre en las hojas a través de los estomas, unos poros ubicados en la superficie de las hojas, por las cuales entra el dióxido de carbono necesario para realizar la fotosíntesis y sale oxígeno y vapor de agua, producto de este mismo proceso.

Los estomas tienen la capacidad de abrirse y cerrarse de acuerdo con las necesidades de dióxido de carbono que tenga la planta, y según las condiciones ambientales, como la intensidad de la luz, la temperatura o el viento. Por lo general, los estomas de las plantas están cerrados en la noche y abiertos en el día.

26. Según lo expuesto en el texto, es correcto afirmar que

a. Los estomas se abren de manera independiente de las necesidades de la planta y las condiciones del ambiente.

b. Los estomas permanecen cerrados durante el día y abiertos en la noche.

c. Las hojas constituyen los órganos fundamentales mediante los cuales las plantas realizan el intercambio gaseoso.

d. Las hojas son los órganos de las plantas que solo permiten llevar a cabo el proceso de la fotosíntesis.

27. Para llevar a cabo el proceso de la fotosíntesis, es necesario que

a. Ingrese oxígeno a la planta y salga dióxido de carbono.

b. Ingrese vapor de agua y salga dióxido de carbono.

c. Ingrese vapor de agua y salga oxígeno.

d. Ingrese dióxido de carbono y salga oxígeno.

28. En relación con el comportamiento de los estomas, se puede concluir que estos

a. Se abren en presencia de luz.

b. Se abren en ausencia de luz.

c. Permanecen cerrados.

d. Permanecen abiertos.

29. Las diatomeas, algas microscópicas fotosintetizadoras, que se fijan a sustratos están favorecidas para su crecimiento en limos o rocas, permitiéndoles

a. Captar la mayor cantidad de luz por su condición autótrofa.

b. Obtener la materia orgánica disponible para su descomposición.

c. Consumir otros microorganismos presentes en el agua.

d. Adherirse a los peces para desplazarse a favor de la corriente.

30. Seleccione las palabras adecuadas que completarían el siguiente texto: 
En la respiración se toma aire rico en y se elimina aire rico en
a. Nitrógeno y aire
b. Oxígeno y dióxido de carbono
c. Nutrientes y desechos
d. Desechos

¡Muchas gracias por su participación! 